\title{
Étude Des Aspects Épidémiologiques Du Choléra Dans Le District De Santé De Tcholliré (Nord- Cameroun)
}

\author{
Justin Ndié, Inf. - Epidemiologist, Msc in Public Health \\ Ministry of Public Health, Cameroon \\ Isaac Bayoro, MD of Public Health \\ Pitoa Health District, North-Cameroon \\ Isidore Takoukam, State Registered Nurse \\ Tcholliré Health District, North-Cameroon \\ Paul Wina, Assistant Technician of Laboratory \\ Tcholliré District Hospital, North-Cameroon
}

doi: 10.19044/esj.2016.v12n15p278 URL:http://dx.doi.org/10.19044/esj.2016.v12n15p278

Abstract

Cholera evolves in an endemo-epidemic mode in developing countries. In Cameroon, because of the weakness of our health system, mainly of the epidemiologic surveillance and inadequate timely response, many localities in our country are faced with intermittent cholera epidemics. This study was aimed at analyzing the epidemiological aspects of cholera in the Tcholliré Health District (North Region-Cameroon) in 2011. We therefore applied a retrospective cross-sectional study using data on cholera cases registered within the $27^{\text {th }}$ and the $40^{\text {th }}$ epidemiological weeks in the Tcholliré Health District in 2011. All patients infected with cholera were included. The demographic, clinical and therapeutic variables, as well as the origin and the evolution of cases were drawn from the cholera data base of the Tcholliré Health District. Data analysis was done using SPSS and Excel software. Between the $27^{\text {th }}$ and $40^{\text {th }}$ epidemiological weeks, 334 cases of cholera were notified in the Tcholliré Health District. The sex ratio was 1.27 $(187 \mathrm{M} / 147 \mathrm{~W})$, the men were most infected (56\%). The mean age was $28 \pm 17$ years, youths aged 16 to 34 were the age group that were most affected with $42.20 \%$. The number of cases varied significantly based on the sex ratio and the health area $(\mathrm{p}=0.012)$. The rate of relapse was $0.61 \%$ and the mortality rate was $1.2 \%$. All the patients had watery stools and vomiting. In addition, all patients were treated primarily with intravenous normal saline at $0.9 \%$ or Ringer Lactate and ORS for oral therapy. The antibiotic used was Doxycycline $100 \mathrm{mg}$. Cholera remains a diarrheal disease that threatens all 
regions in Cameroon. It comes about as a result of a deficiency in individual and collective hygienic practices, and a passive epidemiologic monitoring. Nevertheless, the institution of a monitoring system and also effective combat methods will significantly reduce the recurrence of cholera epidemics.

Keywords: Cholera, epidemiological monitoring, epidemiological week, Tcholliré Health District, North Region-Cameroon.

\section{Résumé}

Le choléra évolue en mode endémo-épidémique dans la quasi-totalité des pays en développement. Au Cameroun, à cause de la faiblesse du système de santé, principalement de la surveillance épidémiologique et de l'inadéquat timing de réponse, de nombreuses localités du pays font face de façon intermittente aux épidémies de choléra. Cette étude visait à analyser les aspects épidémiologiques de la flambée de choléra de 2011 dans le District de Santé de Tcholliré. Cette étude transversale rétrospective a été menée à partir des données collectées sur les cas de choléra enregistrés en 2011 entre la semaine épidémiologique 27 et 40 dans le District de Santé de Tcholliré. Étaient inclus dans cette étude, tous les patients infectés du choléra. Les variables démographiques, cliniques et thérapeutiques, la provenance et l'évolution des cas ont été extraites de la base des données du choléra du District de Santé de Tcholliré. L’analyse des données a été faite sur les logiciels SPSS et Excel. Entre la semaine épidémiologique 27 et 40, 334 cas de choléra ont été notifiés dans le District de Santé de Tcholliré. Les hommes étaient majoritairement atteints (56\%) avec un sex-ratio de 1,27 $(187 \mathrm{H} / 147 \mathrm{~F})$. L'âge moyen était de $28 \pm 17$ ans, les jeunes âgés de 16 à 34 ans étaient les plus touchés avec $42,20 \%$. Le nombre de cas variait significativement selon le sexe et les aires de santé $(p=0,012)$. Le taux de rechute était de $0,61 \%$ et le taux de létalité était de $1,2 \%$. Tous les patients présentaient une diarrhée aqueuse et des vomissements. La prise en charge avait été faite essentiellement par réhydratation intraveineuse au soluté de chlorure de sodium à $0,9 \%$, au Ringer Lactate et aux sels de réhydratation par voie orale (SRO). L'antibiotique utilisé était la doxicycline $100 \mathrm{mg}$. Le choléra demeure une maladie diarrhéique menaçante pour toutes les régions du Cameroun. Elle est consécutive à la fois à une déficience des mesures d'hygiène individuelle et collective et à une surveillance épidémiologique passive. Toutefois, la mise en œuvre d'un système de surveillance et des méthodes de lutte efficace permettra réduire significativement la recrudescence des épidémies de choléra. 
Mots-clés : Choléra, semaine épidémiologique, surveillance épidémiologique, District de Santé de Tcholliré, Région du Nord-Cameroun.

\section{Introduction}

Le choléra est une maladie diarrhéique due à l'infection par la bactérie vibrio cholerae. Il évolue soit sur un faciès épidémique, soit endémo-épidémique dans différentes régions du monde constituant un problème majeur de santé publique (Assogba et al., 2012 ; Pichard et al., 2002). En effet, depuis 2000, l'incidence du choléra a progressivement augmenté avec un nombre de cas cumulé majoré de 43\% (Paraïso et al., 2015). Cependant, en l'absence de traitement, on peut mourir en quelques heures (OMS, 2015).

Chaque année, le choléra affecte plus de 3,5 millions d’individus avec entre 100000 et 130000 décès (Kuma et al., 2014). Ainsi, en 2009, 45 pays ont notifié 221226 cas de choléra, dont 4348 décès soit une létalité de 2,24\% parmi lesquels 1902 cas en Asie et 1897 cas en Océanie (OMS, 2009). En 2011, 58 pays avaient rapporté 589854 cas de choléra avec 7816 décès soit une létalité de 1,33\% (Paraïso et al., Op. cit. ; Dunoyer, 2013) ; en 2013, 47 pays ont déclaré un total de 129064 cas, dont 2102 décès (OMS, 2015). Cependant, en 2014 on estimait son bilan réel entre 3 et 5 millions de cas avec 100000 à 120000 décès par an (OMS, 2014 ; cité par Paraïso et al., 2015). Cet état de chose indique l'incidence et la létalité croissantes du choléra au cours du temps. Pourtant, la qualité de la riposte conditionne l’évolution d’une épidémie et sa létalité (Makoutodé et al., 2010).

L’Afrique reste le continent le plus touché avec plus de $90 \%$ du nombre total mondial (Yatala Tambwe, 2014). Cette situation est particulièrement critique dans la plupart des pays d'Afrique subsaharienne où la maladie sévit sur les modes endémiques et épidémiques, avec des taux de mortalité encore trop élevés malgré les efforts engagés aux niveaux nationaux et internationaux (Assogba et al., 2012). Par ailleurs, le changement climatique global contribue largement à la diffusion du choléra (Tiekoura Konan et al., 2010).

Le Cameroun n’échappe pas à ce fléau. En effet, depuis plus de deux décennies, on enregistre de façon saisonnières des flambées dans différentes régions du pays. En 2009, dans la Région de l'Extrême-Nord, 396 malades ont été enregistrés avec 46 cas de décès soit une létalité de 11,6\% (Koutouan et al., 2011). En 2010, malgré la réactivation des comités régionaux de lutte contre les épidémies créés en 1979 par le gouvernement du Cameroun, un total cumulé de 10759 cas dont 657 décès ont été enregistrés dans 67 districts de santé sur 179 avec une létalité globale de 6,11\%. Cependant, les régions septentrionales restaient les plus touchées avec près de $90 \%$ des cas soit 9404 cas avec 601 décès soit une létalité de 6,39\%. Bien plus, au fil des années, 
l'incidence et la létalité attribuables au choléra reste sans cesse élevées. Ainsi, en 2011, 23152 cas dont 843 décès ont été notifiés essentiellement dans sept des dix régions du pays (MINSANTE, 2011). En 2014, 3355 cas ont été notifiés avec 185 décès soit une létalité de 5,48\% (OMS, 2015).

En 2011, le District de Santé de Tcholliré tout comme les autres District de Santé de la Région du Nord et d'ailleurs, a connu entre la $27^{\text {ième }}$ et la 40 ième semaine épidémiologique une flambée dont le foyer était essentiellement les zones d'orpaillage. Cette situation au demeurant, était consécutive à la mobilité spatiale des populations, particulièrement dans les sites d'orpaillage de Djaba, Mbouckma et Fimbé. En effet, il s'agissait des zones où l'insalubrité et la promiscuité étaient récurrentes, les sources d'approvisionnement en eau potable absentes et les conditions d'hygiène précaires. Ainsi, analyser les aspects épidémiologiques du choléra dans le District de Santé de Tcholliré en 2011 à travers les caractéristiques individuelles, cliniques et thérapeutique des patients s’avérait nécessaire.

\section{Matériels et méthodes}

Cette étude était transversale rétrospective menée sur les données collectées sur les cas de choléra enregistrés entre la $27^{\text {ième }}$ et $40^{\text {ième }}$ semaine épidémiologique (du 05 juillet au 05 octobre 2011) dans le District de Santé de Tcholliré correspondant à 14 semaines épidémiologiques. Étaient inclus dans cette étude, tous les patients infectés du choléra. Les variables démographiques, cliniques et thérapeutiques, la provenance et l'évolution des cas ont été extraites de la base de données du choléra du District de Santé de Tcholliré. L'analyse des données a été faite sur les logiciels SPSS version 22 et Excel. Le test de khi 2 a permis de mesurer l'association entre les variables au seuil de significativité à $5 \%$ et le $\mathrm{V}$ de Cramer variant entre [01] a permis de mesurer la force de l'association. 


\section{Résultats}

\section{Caractéristiques des cas et évolution du choléra}

Tableau 2: Répartition des patients selon leurs caractéristiques

\begin{tabular}{|c|c|c|c|c|c|}
\hline \multirow{2}{*}{ Aires de Santé } & \multirow[b]{2}{*}{ Tcholliré n(\%) } & \multirow[b]{2}{*}{ Kali n(\%) } & \multirow[b]{2}{*}{ Djaba n(\%) } & \multirow[b]{2}{*}{ Total n(\%) } & \multirow[b]{2}{*}{ p-value } \\
\hline & & & & & \\
\hline \multicolumn{6}{|l|}{ Sexe } \\
\hline Masculin & $169(78,9)$ & $6(8,20)$ & $12(12,9)$ & $187(56)$ & \multirow{3}{*}{0,012} \\
\hline Féminin & $116(90,40)$ & $12(3,20)$ & $19(6,40)$ & $147(44)$ & \\
\hline Total & $285(85,30)$ & $18(5,40)$ & $31(9,30)$ & 100 & \\
\hline \multicolumn{6}{|l|}{ Tranche d'âge } \\
\hline$\leq 5 \mathrm{ans}$ & $31(61,60)$ & $4(10,50)$ & $3(7,9)$ & $38(11,4)$ & \multirow{5}{*}{0,75} \\
\hline$[6-15]$ ans & $33(82,50)$ & $1(2,50)$ & $6(15)$ & $40(12,0)$ & \\
\hline [16 - 34] ans & $120(85,10)$ & $8(5,70)$ & $13(9,20)$ & $141(42,2)$ & \\
\hline$[34-44]$ ans & $56(88,90)$ & $2(3,20)$ & $5(7,90$ & $63(18,9)$ & \\
\hline$\geq 45$ ans & $45(86,50)$ & $3(5,80)$ & $4(7,70)$ & $52(15,6)$ & \\
\hline Total & $285(85,30)$ & $18(5,40)$ & $31(9,30)$ & 100 & \\
\hline \multicolumn{6}{|l|}{ Statut } \\
\hline Guéri & $281(87,50)$ & $17(5,20)$ & $30(9,10)$ & $328(98,2)$ & \multirow{4}{*}{0,29} \\
\hline Décédé & $2(50)$ & $1(25)$ & $1(25)$ & $4(1,2)$ & \\
\hline Rechute & $2(100)$ & $0(0,00)$ & $0(0,00)$ & $2(0,6)$ & \\
\hline Total & $285(85,30)$ & $18(5,40)$ & $31(9,30)$ & 100 & \\
\hline
\end{tabular}

Parmi les 334 cas de choléra enregistrés dans le District de Tcholliré en cette période, les hommes étaient les plus touchés (56\%) contre $44 \%$ de femmes avec un sex-ratio de 1,27 (187H/147F). L'âge moyen était de $28 \pm 16$ ans, les jeunes âgés de 16 à 34 ans étaient la tranche d'âge la plus touchée avec $42,2 \%$. Le nombre de cas variait significativement selon le sexe et les aires de santé $(\mathrm{p}=0,012)$. Ainsi, l'aire de santé de Tcholliré avait notifié le plus grand nombre de cas [285(85,3\%); $\mathrm{p}=0,012]$. La majorité des cas (98,8\%) avait trouvé la guérison parmi lesquels seulement 2(0,6\%) avaient eu la rechute. La létalité globale était de 1,2\% (tableau 1).

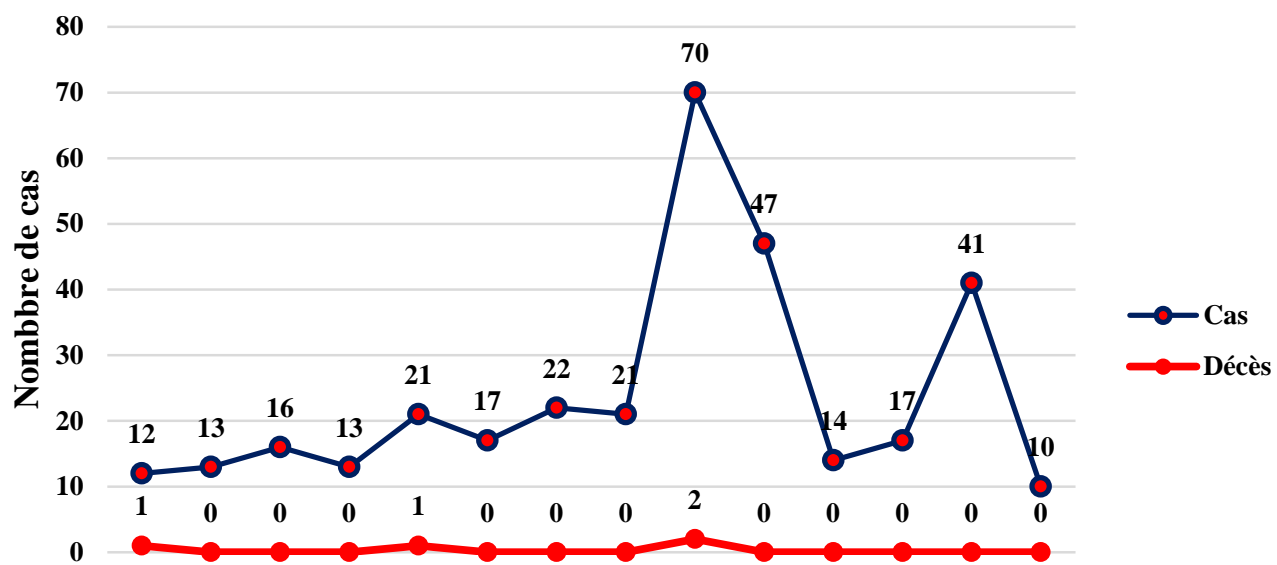

SE 27SE 28SE 29SE 30SE 31SE 32SE 33SE 34SE 35SE 36SE 37SE 38SE 39SE 40

Semaines épidémiologiques

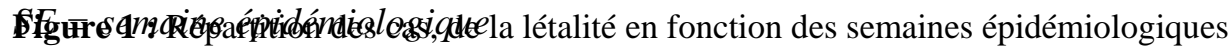


L’épidémie s'était étalée sur 14 semaines, principalement entre la $27^{\text {ième }}$ et la $40^{\text {ième }}$ semaine épidémiologique. Trois pics de fréquence minimale de 41 cas chacun, avaient été observés lors de la $35^{\text {ième }}, 36^{\text {ième }}$ et la $39^{\text {ième }}$ semaine épidémiologique. Cependant, une diminution des cas dès la quarantième semaine avait noté, seulement 10 cas avaient été enregistrés pendant cette semaine (Figure 1).

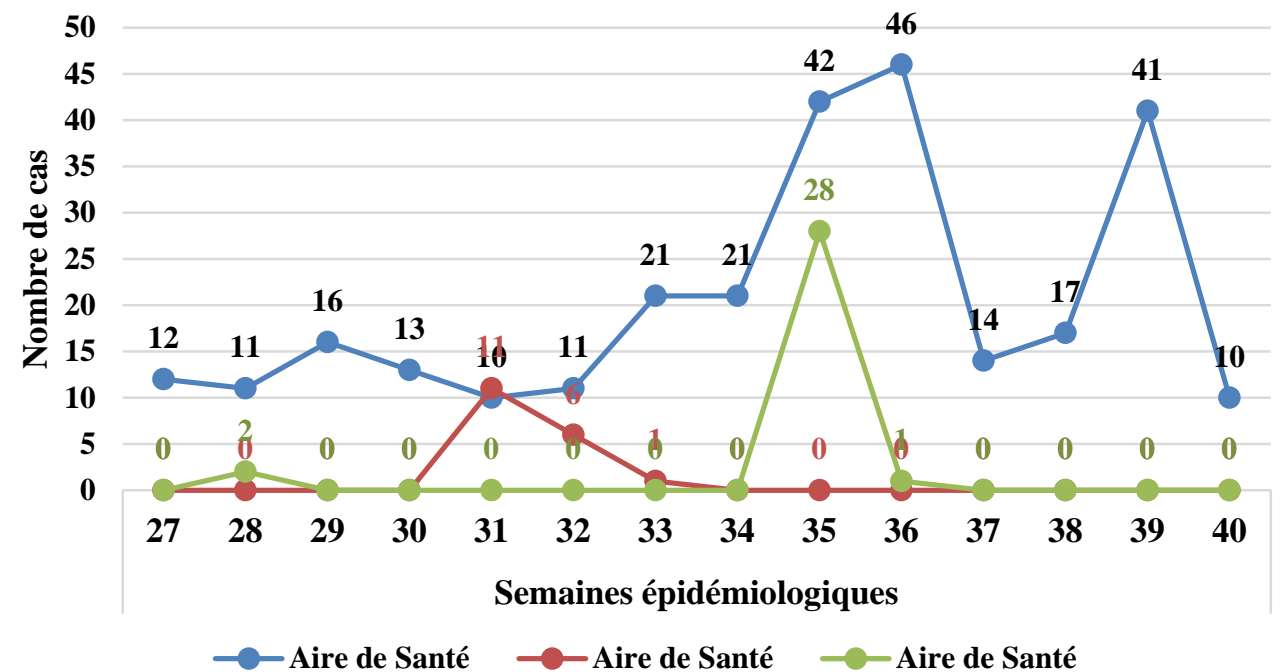

Figure 2 : répartition des cas par des aires de Santé du District de Santé de Tcholliré en fonction des semaines épidémiologiques

L'aire de santé de Tcholliré était la seule ayant notifié des cas pendant les 14 semaines de la flambée du choléra avec trois pics aux semaines épidémiologiques 35, 36 et 39. L’aire de Djaba avait connu un pic à la semaine 35 .

\section{Données cliniques et thérapeutiques}

Tous les patients présentaient une diarrhée aqueuse profuse et des vomissements. La prise en charge avait été faite essentiellement par réhydratation intraveineuse au soluté de chlorure de sodium à $0,9 \%$, au Ringer Lactate et au SRO par voie orale. Le traitement antibiotique était à base de la doxicycline $100 \mathrm{mg}$.

\section{Discussion}

Les hommes étaient majoritairement atteints (56\%) contrairement aux femmes (44\%). Bita Fouda et al. (2012) à Douala, Kyelem et al. (2011) au Burkina Faso et Touré Mariam (2013) dans le district sanitaire de Mopti au Mali avaient trouvé les résultats similaires. Cette prédominance masculine 
apparait liée au fait que les hommes étaient les plus actifs et les plus nombreux dans les zones à risques et les foyers d'épidémies (Bouckma, Tcholliré, Djaba).

Les jeunes âgés de 16 à 34 ans étaient la tranche d'âge la plus touchée avec 42,2\% comme dans les études de Bita Fouda et al. (2012) à Douala, de Patassi et al. (2009) à Lomé au Togo. Cette fréquence élevée chez cette frange de la population s'explique par le fait qu'ils étaient majoritairement nombreux, mais les plus enclins à la promiscuité, à l’insalubrité et au contact avec les cas malades de par leur dynamisme. Par contre, Morillon et al. (2002) avaient trouvés à Djibouti que les enfants âgés de 0 à 5 ans étaient les plus atteints. Aussi, Kyelem et al. (2011) au Burkina Faso avaient trouvé que les jeunes de 20 à 39 ans (51,1\%) étaient les plus touchés.

À propos de la zone affectée, l'aire de santé de Tcholliré avait rapporté le plus grand nombre de cas $(85,3 \%)$. Cette forte concentration des cas dans l'aire de Tcholliré s'explique par le fait que l'aire était une zone urbaine cosmopolite avec une forte mobilité des populations. Bien plus, cette aire de santé était ouverte et était à proximité géographique des zones d'orpaillage d'où la possibilité d'importation du choléra. Ces résultats corroboraient ceux de Kyelem et al. (2011) au Burkina Faso et Touré Mariam (2013) à Mopti qui rapportaient que des patients de choléra provenaient du milieu urbain. L'épidémie avait connu trois pics aux semaines épidémiologiques 35, 36 et 39. Ces pics correspondaient aux périodes de pluviométries élevées, périodes pendant lesquelles il y a une forte circulation du vibrion cholérique.

Le taux de létalité était élevé (1,20\%) contrairement à celui préconisé par l'OMS (2006) qui doit être en dessous de 1\%. Ces résultats corroboraient ceux de Bita Fouda et al. (2012) qui avaient trouvé un taux de létalité de $1,27 \%$ à Douala. Cette situation s'explique par l'arrivée tardive des cas dans les services de soins. Aussi, par le fait de l'enclavement de certains foyers épidémiques (Bouckma, Fimbé, Mayo-Sala) entrainant de l'accès difficile. Par ailleurs, nos résultats étaient nettement supérieurs à ceux de Makoutodé et al. (2010) au Bénin (4,8 fois) et de Touré Mariam (2013) à Mopti (1,33 fois) qui rapportaient une létalité respective de $0,25 \%$ et de $0,9 \%$.

Tous les patients avaient présenté une diarrhée aqueuse et des vomissements. La prédominance de ces signes et manifestations traduisait de fait, la symptomatologie classique du choléra. Ces résultats corroboraient ceux de Kyelem et al. (2011) au Burkina Faso et de Touré Mariam (2013) à Mopti qui révélaient la même symptomatologie.

La prise en charge était faite au soluté de chlorure de sodium à 0,9\%, au Ringer Lactate et au SRO par voie orale et l'antibioprophylaxie était à base de la doxicycline $100 \mathrm{mg}$ malgré l'isolement de la souche responsable de la flambée et l'effectivité de l'antibiogramme. Nos résultats sont 
comparables à ceux de Makoutodé et al. (2010) au Bénin. D’autre part, Bita Fouda et al. (2012) à Douala constataient que le vibrio cholerae était sensible à la Doxycycline et l'Érythromycine. Par contre, au Burkina Faso, Kyelem et al. (2011) signalaient que les vibrions cholériques étaient très sensibles à la ceftriaxone, à la ciprofloxacine, l'acide nalidixique et la doxycycline.

\section{Conclusion}

Le choléra est une maladie qui n’épargne aucune classe sociale, davantage favorisé par le faible respect des mesures d'hygiène individuelle et collective. La recrudescence des flambées met à jour le problème du faible accès des populations à l'eau potable, de leur faible utilisation des latrines, de la promiscuité et certaines pratiques traditionnelles mais surtout des stratégies ponctuelles de lutte mise en œuvre par les pouvoirs publics et la faiblesse du système de surveillance épidémiologique sans ignorer la grande mobilité des populations et les saisons de pluies. L’épidémie de choléra au District de Santé de Tcholliré en 2011 était plus ou moins grave et les jeunes âgés de 16 à 34 ans étaient les plus touchés avec 42,2\%. Partant, la mise en œuvre des mesures pérennes de promotion de l'hygiène individuelle et collective, la surveillance de cette maladie, davantage le renforcement du système d'approvisionnement en eau potable surtout dans les zones à risques par le Ministère de la Santé Publique en partenariat avec les autres départements ministériels restent les moyens optimaux de prévention cette maladie à potentiel épidémique. De plus, la prise en charge médicale et communautaire précoce du choléra permet de réduire sa létalité.

\section{References:}

Assogba A. L. et al. (2015). L'initiative contre les maladies diarrhéiques et entériques en Afrique : une contribution à la lutte contre le choléra. Médecine d'Afrique noire. ISSN 0465-4668. vol. 59, $n^{\circ} 5$, pp. 251-257.

Bita Fouda, A. A. et al. (2012). Épidémie de choléra à Douala en 2011 épidémiologie, clinique et bactériologie. Revue de Médecine et de Pharmacie. Vol. 2, $n^{\circ}$ 1. Pp. 113-120.

Dunoyer, E. (2013). Lutter contre le choléra ! le rôle des secteurs EAH et SMPS dans la lutte contre le choléra. ACF-International. Manuel Pratique. Action contre la Faim-France.

Koutouan et al. (2011). Rapport d'évaluation : Analyse de la situation du choléra Cameroun. Africhol. Agence de Médecine Préventive. Rapport visite pays Cameroun_V. 23/03/2011.

Kuma et al. (2014). A laboratory-based surveillance assessment for epidemic vibrio cholerae 01 in greater Accra Region, Ghana-2013. African Journal for Epidemiology. ISSN 2384-5694. June 2014. Vol.2. supp. 1. 
Kyelem, C. G. et al. (2010). Épidémie de choléra au Burkina Faso en 2005 : aspects épidémiologiques et diagnostiques. The Pan African Medical Journal. ISSN 1937-8688.

Makoutodé, M. et al. (2010). La riposte à l'épidémie de choléra de 2008 à Cotonou (Bénin). Santé Publique 2010/4. Vol. 22. Pp. 425-435.

Morillon, M. et al. (2002). Une épidémie de choléra à Djibouti (mai 2000 janvier 2001). Med Trop. (Mars). 62(5). Pp. 497-502.

OMS. (2006). Flambées de cholera : évaluation des mesures mises en œuvre en cas de flambée et amélioration de la préparation. Genève. WHO/CDS/CPE/ZFK/2004.4.

OMS. (2010). Rapport annuel. Bureau Pays du Cameroun. Yaoundé Cameroun

OMS. (2015). Choléra. Aide-mémoire N¹07. consulté le 16 mars 2016 Sur www.who.int/mediacentre/factsheets/fs107/fr/.

OMS. (2015). Relevé épidémiologique hebdomadaire. Sw.fr./Fr.s. 54610.2015. ISSN 0049-8114. $n^{\circ}$ 40. 2015. 90. Pp. 517-544.

Paraïso et al. (2015). Factors associated to populations’ behaviour towards cholera in Cotonou (Benin). Int. J. Biol. Chem. Sci. 9(2): 710-722, 2015. ISSN 1997-342X (Online), ISSN 1991-8631 (Print). DOI : http://dx.doi.org/10.4314/ijbcs.v9i2.12.

Patassi et al. (2009). Épidémie de choléra à Lomé (Togo) : aspects épidémiologiques, cliniques et évolutifs. Journal de la Recherche Scientifique de l'Université de Lomé. ISSN : 1027-1988.

Pichard, E. et al. (2002). Malintrop Afrique: Manuel de maladies infectieuses pour l'Afrique. Éditions John Libbey Eurotext. ISBN 2-74200372-X. Paris.

Tiekoura Konan, B. et al. (2010). Caractérisation Moléculaire des Souches de Vibrio Cholerae Non O1, Non O139 Isolées des Eaux Lagunaires de Grand-Lahou (Côte d'Ivoire). European Journal of Scientific Research. ISSN 1450-216X Vol. 45 No. 3, pp.333-345.

Touré Mariam O. Sao (2013). Épidémiologie du Choléra dans le district sanitaire de Mopti en 2011. Thèse de doctorat de Médecine. Université des Sciences Techniques et de Technologies de Bamako. République du Mali.

Yatala Tambwe, N. (2014). Évaluation de niveau de connaissance des femmes de l'aire de sante de Kituku sur les voies de contamination de maladies diarrhéiques In Revue Université Sans Frontières pour une Société Ouverte de l'université Distant Production House. ISSN 2313-285X. Vol. 2. PP. 128-132. 